\title{
Zur Bedeutung des Sonnenhormons Vitamin D in der Onkologie - ein Update
}

\author{
Jörg Spitz
}

\section{Zusammenfassung}

Aus dem Knochen-Vitamin D mit seinem begrenzten Aufgabenbereich im Kalziumstoffwechsel ist innerhalb weniger Jahre das Sonnenhormon geworden, das für eine regelrechte Funktion nahezu aller Organe und Zellen unseres Körpers benötigt wird. Unter anderem werden mehr als 2000 Gene von Vitamin D beeinflusst. Die eigentlich problemlose Synthese mit Hilfe der UVB-Strahlung der Sonne in der Haut wird durch mehrere Aspekte unseres modernen Lebensstils behindert, sodass sich die Mehrheit der Bevölkerung in einer Mangelsituation befindet. Damit ist Vitamin D an der Entstehung/ Vermeidung nahezu aller chronischen Erkrankungen und damit auch am Krebsgeschehen im Körper beteiligt. Das Sonnenhormon bewirkt eine Unterdrückung des Tumorwachstums, eine Abschwächung der Signale zur Metastasierung, eine gesteigerte Veranlassung des Zelltodes (Apoptose) und eine Differenzierung der Zellen (in Richtung Gutartigkeit) sowie die Reduzierung der Gefäßneubildung durch die Tumoren. Dies gilt auch und insbesondere für zwei sehr häufige Tumorarten: den Brustkrebs der Frau und das Kolonkarzinom bei beiden Geschlechtern. Hier zeigen sowohl epidemiologische als auch prospektive Untersuchungen ein Vitamin-D-abhängiges Risiko für die Tumorentstehung von $50-70 \%$. Die Risikoreduktion für die Wahrscheinlichkeit, an einem solchen Tumor zu versterben, liegt in einer vergleichbaren Dimension. Es erscheint daher angezeigt, einen ausreichenden Vitamin-D-Spiegel (>30ng/ml) sicherzustellen, um die positiven Effekte des Sonnenhormons nicht nur für den Knochenstoffwechsel, sondern auch für das Tumorgeschehen im Körper zu gewährleisten.

Schlüsselwörter: Vitamin D, Sonnenhormon, endokrine Funktion, Wirkung bei Krebserkrankungen, Kolonkarzinom, Mammakarzinom

\section{Einleitung}

Die neue, erweiterte Bedeutung des Sonnenhormons ergibt sich daraus, dass diese Substanz nicht mehr als Vitamin, sondern als Vorstufe eines Steroidhormons oder sogar als eigenständiges Steroidhormon angesehen wird. Es wird im Körper in Kooperation von Leber und Haut unter dem Einfluss von Sonnenlicht (UVB-Anteil) aus Cholesterol synthetisiert. Daher resultiert auch die ergänzende Bezeichnung „Sonnenhormon“. Die Haut wird damit zu einer Drüse, einem endokrinen Organ wie die Schilddrüse oder die Keimdrüsen. Ferner ist der Wirkungsbereich nicht auf den Knochenstoffwechsel limitiert. Nahezu alle Körperzellen verfügen über Rezepto- ren für Vitamin D. Hier wird es für die Regulation zahlreicher intrazellulärer Prozesse, unter anderem für die Steuerung von mehr als 2000 Genen benötigt. Das sind rund $10 \%$ des menschlichen Genoms [9].

Leider steht diesem neu erkannten ubiquitären Einsatz im menschlichen Körper die ebenfalls neue Tatsache gegenüber, dass nicht nur die Säuglinge und alte Menschen, sondern nahezu die gesamte Bevölkerung von einem Mangel an Vitamin D betroffen ist. Diese zunächst unglaubwürdig klingende Feststellung wird nicht nur durch eigene, sondern auch durch zahlreiche internationale Studien gestützt, wobei selbst sonnenverwöhnte Länder wie die Türkei, Indien oder Australien keine Aus- nahme machen. Der Grund für den Vitamin-D-Mangel ist nicht mehr (vorwiegend) der Breitengrad mit einer im Winter tief stehenden Sonne, deren UVB-Strahlung keine Kraft mehr für die Vitamin-DSynthese in unserer Haut hat, sondern unser Lebensstil: wir halten uns überwiegend in Räumen und Fahrzeugen auf, tragen verhüllende Kleidung und decken die noch herausschauenden Hautpartien mit Sonnenschutzmitteln ab. Da hat die Sonne keine Chance mehr, eine ausreichende Vitamin-D-Produktion zu bewirken.

Durch das Zusammentreffen von einem breiten Aufgabenkatalog in unserem Körper und einer ausgedehnter Mangelsituation in der Bevölkerung ist Vitamin $\mathrm{D}$ an der Entstehung/Vermeidung nahezu aller chronischen Erkrankungen und damit auch am Krebsgeschehen im Körper beteiligt.

Liste der mit Vitamin D assoziierten Eigenschaften (Auszug):

- senkt den Blutdruck

- fördert das angeborene und erworbene Immunsystem

- produziert körpereigene Antibiotika (AMP)

- schützt die Nervenzellen (z.B. vor MS)

- fördert eine Schwangerschaft und schützt ihren Verlauf

- verbessert die Überlebensrate von KHK-Patienten

- reduziert das Risiko für Diabetes Typ I und Typ II

- schützt vor peripherer arterieller Verschlusserkrankung

- kräftigt die Muskulatur und verzögert die Pflegebedürftigkeit im Alter 


\begin{tabular}{|c|c|c|c|}
\hline Phase & Diagramm & Prozess & Aktion von Vitamin D \\
\hline $\begin{array}{l}\text { Vitamin D regel- } \\
\text { recht, Speicher } \\
\text { aufgefüllt }\end{array}$ & & $\begin{array}{l}\text { Verbindungen und } \\
\text { Kommunikation zwischen den } \\
\text { Zellen intakt, Wachstumshem- } \\
\text { mung bei Kontakt aktiv, geringe } \\
\text { Zellteilungsrate, normale Steue- } \\
\text { rung der Apoptose }\end{array}$ & $\begin{array}{l}\text { Vitamin-D-Spiegel zwischen } \\
40-60 \mathrm{ng} / \mathrm{ml} \text {; die links stehenden } \\
\text { Prozesse werden durch lokale } \\
\text { Biosynthese von } 1,25-\mathrm{OH} \text {-Vita- } \\
\text { min D aufrechterhalten }\end{array}$ \\
\hline $\begin{array}{l}\text { 1. Trennung } \\
\text { Bedingt durch nied- } \\
\text { rige Vitamin-D- und } \\
\text { Kalziumspiegel }\end{array}$ & & $\begin{array}{l}\text { Die Zellen rücken leicht auseinan- } \\
\text { der; Zellverbindungen, Kommuni- } \\
\text { kation und Wachstumshemmung } \\
\text { bei Kontakt sind reduziert }\end{array}$ & Förderung der Zellverbindungen \\
\hline 2. Beginn & & $\begin{array}{l}\text { Auftreten von Störungen in der } \\
\text { DNA oder der epigenetischen } \\
\text { Steuerung, wodurch die Teilungs- } \\
\text { rate von Epithelzellen gesteigert } \\
\text { wird }\end{array}$ & $\begin{array}{l}\text { Förderung der Zellverbindungen } \\
\text { und der Wachstumshemmung } \\
\text { bei Kontakt, Absenkung der } \\
\text { Teilungsrate }\end{array}$ \\
\hline $\begin{array}{l}\text { 3. Natürliche } \\
\text { Selektion }\end{array}$ & & $\begin{array}{l}\text { Rasche Teilung und aggressi- } \\
\text { ve Vermehrung dieser Zellen } \\
\text { überwiegen; ein } 2 \% \text { schnelleres } \\
\text { Wachstum wird nach } 9.000 \\
\text { Teilungen den lokalen Gewebs- } \\
\text { bereich ausfüllen }\end{array}$ & $\begin{array}{l}\text { Verhindert die Teilung reifer Zel- } \\
\text { len, reduziert damit die Chance } \\
\text { der natürlichen Selektion für den } \\
\text { Stamm der sich rasch vermeh- } \\
\text { renden Zellen }\end{array}$ \\
\hline $\begin{array}{l}\text { 4. Überwucherung, } \\
\text { Durchdringen der } \\
\text { Basalmembran }\end{array}$ & & $\begin{array}{l}\text { Rasch vermehrende Zellen } \\
\text { konkurrieren in der Versorgung } \\
\text { mit Nahrung und Blut; die } \\
\text { Basalmembran wird aufgelöst } \\
\text { und durchdrungen }\end{array}$ & $\begin{array}{l}\text { Baut die Verbindung zwischen } \\
\text { den Zellen und die Wachstums- } \\
\text { hemmung bei Kontakt wieder auf }\end{array}$ \\
\hline
\end{tabular}

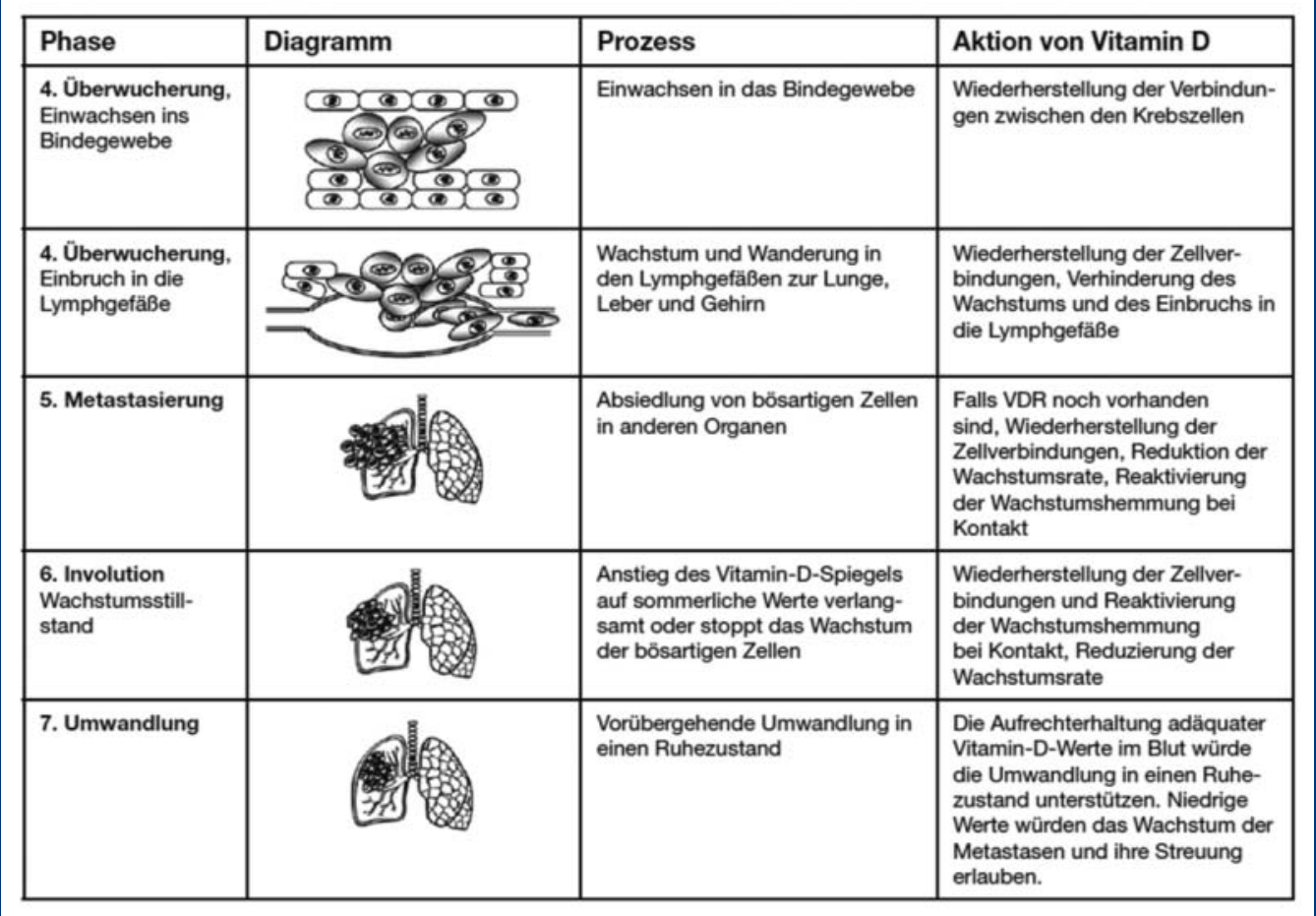

Abb. 1: Schutzfunktion von Vitamin D bei bösartigen Tumorerkrankungen. Das von der Arbeitsgruppe um Garland entwickelte DINOMITModell zur Tumorentstehung (DINOMIT = Disjunction, Initiation, Natural selection, Overgrowth, Metastasis, Involution, and Transition) [6, 26] dokumentiert den Einfluss von Vitamin D in allen Stadien des Tumorgeschehens (mit freundlicher Genehmigung aus [6]).

\section{Allgemeine Eigenschaften von Vitamin D in Bezug auf das Krebsgeschehen}

Inzwischen wurden zahlreiche und unterschiedliche Schutzfunktionen von Vitamin D bei der Entstehung und Ausbreitung der Mehrzahl der bösartigen Tumore nachgewiesen: Das Sonnenhormon bewirkt eine Unterdrückung des Tumorwachstums, eine Abschwächung der Signale zur Metastasierung, eine gesteigerte Veranlassung des Zelltodes (Apoptose) und eine Differenzierung der Zellen (in Richtung Gutartigkeit) sowie die Reduzierung der Gefäßneubildung durch die Tu- moren. Das Ausmaß dieser Schutzfunktion wird in Abb. 1 deutlich, die ein von der Arbeitsgruppe um Garland entwickeltes Modell der Tumorentwicklung enthält [6]. Entscheidend ist dabei die Tatsache, dass Vitamin D von den ersten Störungen im Zellstoffwechsel bis zum Stadium der Metastasierung einen hemmenden Effekt auf 
das Tumorgeschehen hat. Welche weitreichenden Konsequenzen dies für einzelne Tumorarten bedeutet, soll wegen des begrenzten Umfangs dieses Beitrags beispielhaft an zwei Tumorarten erläutert werden, die zu den häufigsten bösartigen Tumoren des Menschen zählen.

\section{Das Mammakarzinom}

Das Mammakarzinom ist nicht nur der häufigste bösartige Tumor des weiblichen Geschlechts, sondern auch prognostisch in vielen Fällen ungünstig. Es stellt daher die zweithäufigste Todesursache bei Frauen dar. Umso eindrucksvoller sind die zahlreichen Publikationen über die Schutzfunktion von Vitamin D bei diesem Tumor. In der Tumorprävention wird eine Risikoreduktion bis zu einem Faktor drei und in der Metastasierung um mindestens die Hälfte erreicht.

Die Arbeitsgruppe von Garland, die sich bereits sehr lange mit dem Thema Vitamin D beschäftigt, fasste die Ergebnisse von zwei Studien mit insgesamt $1.760 \mathrm{~Pa}-$ tientinnen mit einem neu nachgewiesenen Mammakarzinom zusammen [5]. Dabei zeigte die Gruppe mit den höchsten Vitamin-D-Spiegeln (> $120 \mathrm{nmol} / \mathrm{l}$ ) gegenüber den Frauen mit den niedrigsten Vitamin-D-Spiegeln ( $<32 \mathrm{nmol} / \mathrm{l})$ eine $50 \%$ ige Risikoreduktion.

Anfang 2008 veröffentlichte die Arbeitsgruppe um Abbas am Deutschen Krebsforschungszentrum (DKFZ) die Ergebnisse von 1.365 nach den Wechseljahren an Brustkrebs erkrankten Frauen [1]. Wie bei den Studien von Garland konnten bei dieser Untersuchung die Vitamin-DSpiegel im Blut der betroffenen Frauen gemessen werden. Die Ergebnisse wurden in fünf Gruppen mit aufsteigendem VitaminD-Gehalt eingeteilt (Abb. 2). Erneut zeigte sich, dass die Schutzfunktion mit zunehmendem Vitamin-D-Spiegel immer besser wird. Die Risikoreduktion in diesem Kollektiv betrug sogar $69 \%$ ! Anders ausgedrückt könnten 7 von 10 Frauen von einem Mammakarzinom verschont bleiben, wenn ihr Körper über genügend Vitamin D verfügt, um sich selbst vor der Krebserkrankung zu schützen!

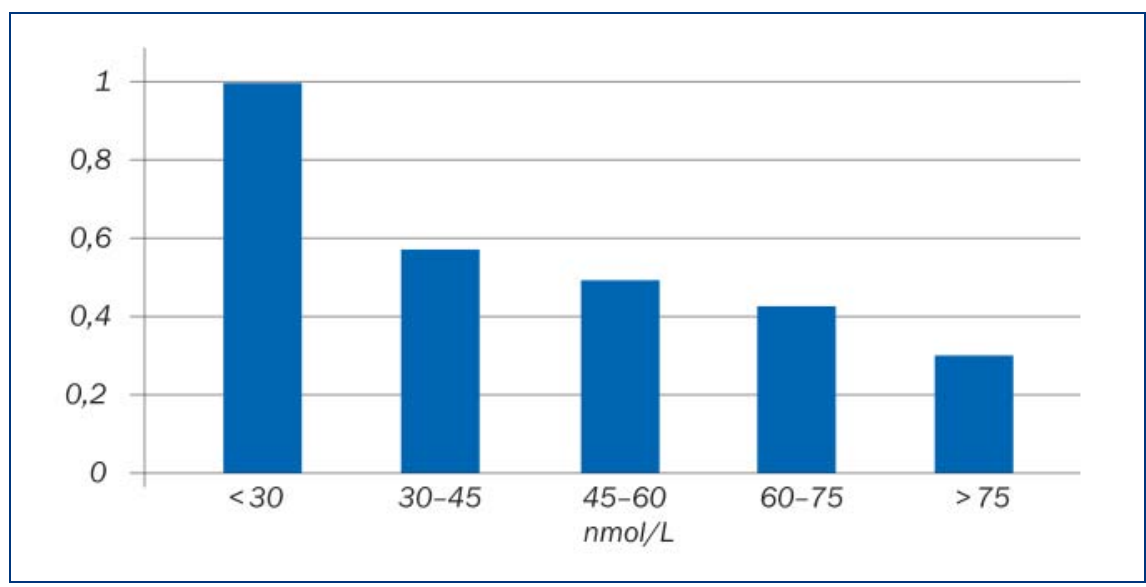

Abb. 2: Sinkendes Risiko für die Ausbildung eines Mammakarzinoms mit steigendem VitaminD-Gehalt im Blut [1].

Aber auch in der Nachsorge von Tumorpatientinnen hat Vitamin D eine besondere Bedeutung. Mitte 2008 berichten kanadische Wissenschaftler auf der Jahrestagung der Amerikanischen Krebsgesellschaft, dass das Sonnenhormon auch vor Metastasen und frühzeitigem Tod durch den Krebs schützt [7]. 512 Frauen wurden 10 Jahre lang nach ihrer Operation wegen eines bösartigen Brusttumors nachuntersucht. Nur 24\% wiesen initial einen ausreichenden Vitamin-D-Spiegel im Serum auf. Im Verlauf führte ein niedriger Vitamin-D-Spiegel zu einer Steigerung des Risikos für eine Metastasierung um 94\% und um $73 \%$ für einen vorzeitigen Tod!

Dieser erste Bericht über die große Bedeutung von Vitamin D in der Nachsorge des Mammakarzinoms wurde 2014 durch eine Meta-Analyse von mehreren Studien bestätigt [21]. Die Berechnung ergab im Mittel eine 60\%ige Absenkung der Mortalität, wenn die Gruppe mit den höchsten Vitamin-D-Spiegeln (Mittelwert etwa 30 $\mathrm{ng} / \mathrm{ml}$ ) mit der Gruppe der niedrigsten Vitamin-D-Spiegel (Mittelwert etwa 17 ng/ $\mathrm{ml}$ ) verglichen wurde. Lediglich eine (relativ kleine) der fünf Studien zeigte keine Risikoreduktion.

Eine französische Studie untersuchte den Zusammenhang zwischen oraler Vitamin-D-Aufnahme, Breitengrad des Wohnortes und täglicher UV-Dosis in Bezug auf das Risiko, einen bösartigen Brusttumor zu entwickeln [3]. Dazu wurden die Daten von 67.000 französischen Frauen im Verlauf von 10 Jahren herangezogen. In die- sem Zeitraum kam es in 2871 Fällen zur Entwicklung eines bösartigen Brusttumors. Die Auswertung ergab keinen signifikanten Einfluss einzelner Faktoren auf das Tumorgeschehen. Das Zusammenwirken der beiden Faktoren südlicher Wohnort plus hohe orale Vitamin-D-Aufnahme ergab jedoch eine Risikoreduktion um 43\%. Die Schlussfolgerung der Autoren lautet, dass es wohl eine Art Schwellendosis gibt, die überschritten werden muss, um den positiven Effekt des Sonnenhormons zu bewirken. Dabei ist es ohne Bedeutung, aus welcher Quelle Vitamin D stammt.

Ein weiterer Aspekt gewinnt zunehmend an Bedeutung. Angesichts seiner vielfältigen Wirkungen in den unterschiedlichsten Geweben des Körpers hat das Sonnenhormon offensichtlich bei den Krebspatienten eine zusätzliche Bedeutung: es schützt vor Nebenwirkungen zahlreicher onkologischer Therapeutika. Dieser Effekt ist jedoch ebenso wie bei der direkten Tumorwirkung von Vitamin D nur gegeben, wenn ein ausreichender Spiegel vorhanden ist. Die nachfolgenden Ausführungen zeigen, wie umfangreich die Palette der beobachteten positiven Wirkungen des Sonnenhormons im Rahmen onkologischer Therapien ist [9].

So konnte bei Brustkrebspatientinnen unter einer Anthrazyklin- und Taxan-haltigen Polychemotherapie ein deutlicher Abfall des 25-OH-D-Spiegels beobachtet werden. Einige Zytostatika (z.B. Cyclophosphamid, Paclitaxel) sind Liganden des 


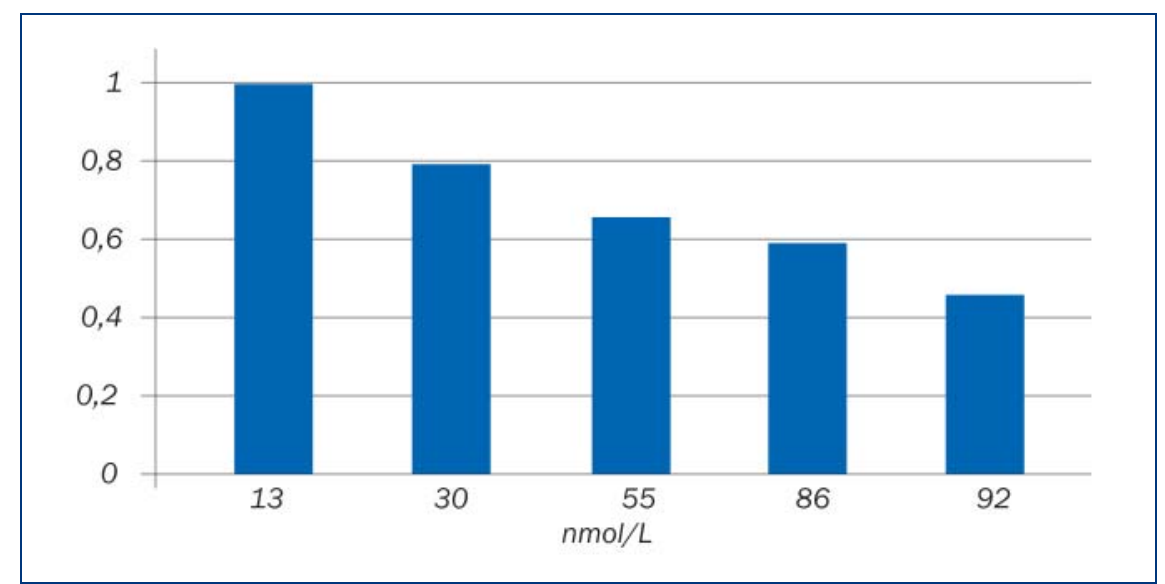

Abb. 3: Abhängigkeit des Darmkrebsrisikos vom Vitamin-D-Spiegel im Blut [8].

Pregnan-X-Rezeptors und können dadurch über die Induktion der 24-Hydroxylase den enzymatischen Abbau von 25-OH-D und 1,25-(OH)2-D im Verlauf der Chemotherapie steigern. Docetaxel ist ein bekannter Auslöser kutaner Nebenwirkungen und Geschmacksstörungen. Ein Vitamin-D-Mangel kann das Auftreten einer Chemotherapie-induzierten Mukositis und Dysgeusie begünstigen. In Fallberichten konnten mukokutane Nebenwirkungen (z.B. Stomatitis) und Geschmacksstörungen, die bei Krebspatienten unter einer Polychemotherapie (Docetaxel, Carboplatin und Trastuzumab) oder FOLFOX6 auftraten, erfolgreich durch die Supplementierung von Vitamin D3 behandelt werden. Auch Arthralgien und Fatigue unter der Therapie mit Aromatasehemmern wie Letrozol konnten durch die labordiagnostisch validierte Supplementierung von Vitamin D (z.B. 50.000 I.E. Vitamin D/Woche für 12 Wochen, p.o.) bei Brustkrebspatientinnen mit Vitamin-D-Mangel deutlich gelindert werden.

\section{Kolonkarzinom}

Bösartige Tumore des Darms sind die zweithäufigste Tumorart bei Frauen und Männern. Ähnlich wie beim Mammakarzinom ist die Prognose nicht günstig, wenn nicht frühzeitig operiert wird. Die in den vergangenen Jahren bundesweit geförderte Darmspiegelung soll dies ermöglichen, wird jedoch von der Bevölkerung nicht in ausreichendem Maße genutzt. Umso wichtiger ist es da, dass die Studien- lage zeigt, dass auch die bösartigen Darmtumore durch Vitamin D in einem ähnlich hohen Maß wie der Brustkrebs positiv beeinflusst werden: Risikoreduktion bis zu $72 \%$, einen Tumor zu entwickeln, und etwa $50 \%$ Reduktion der Gesamtsterblichkeit nach der Operation!

Die Arbeitsgruppe von Garland hat bereits vor 30 Jahren eine erste Arbeit über die bösartigen Darmtumore veröffentlicht. Darin verweisen die Wissenschaftler auf den Zusammenhang zwischen dem Breitengrad, also der Sonnenexposition der Menschen, und dem Auftreten von bösartigen Darmtumoren. Der Anlass für ihre Überlegungen war der damalige Krebsatlas der USA, der im Nordosten im Vergleich zum Südwesten des Landes eine deutlich erhöhte Sterblichkeit an Darmkrebs zeigt.

Im Jahr 2007 veröffentlichte die gleiche Arbeitsgruppe eine Meta-Analyse von fünf Studien über die Wirkung von Vitamin D auf die Entstehung von kolorektalen Tumoren [8]. Wie schon in der Arbeit von Abbas zu den bösartigen Brusttumoren beschrieben, erfolgte die Einteilung der Vitamin-D-Spiegel im Blut in aufsteigenden Gruppen mit den Werten: 15, 30, 55, 86, $92 \mathrm{nmol} / \mathrm{l}$. Als Risikoeinstufung für diese Gruppen resultierten die folgenden Faktoren: $1,00,0,80,0,66,0,59,0,46$. Der Vergleich der Gruppe mit dem höchsten Vitamin-D-Gehalt im Blut zu der Gruppe mit dem niedrigsten Vitamin-D-Spiegel ergab somit eine Reduktion des Risikos, an einem Darmtumor zu erkranken, von 54\% (Abb. 3).
Im gleichen Jahr stellten Freedman und Mitarbeiter die Ergebnisse aus der NHANES-III-Studie in Amerika vor [4]. Dies ist ein weiteres, großes Untersuchungskollektiv, das bereits seit vielen Jahren mit den verschiedensten Fragestellungen untersucht wird. Die hier veröffentlichten Daten beziehen sich auf 16.800 erwachsene Patienten in der Zeit von 1988 bis 2000. Auch bei diesem Projekt war es möglich, Vitamin D im Blut zu untersuchen. Allerdings erfolgte die Sammlung der Proben in den nördlichen Bundesstaaten der USA im Sommer und in den südlichen Staaten im Winter. Der Vergleich der Gruppe mit einem Vitamin-D-Spiegel $>80 \mathrm{nmol} / \mathrm{l}$ zu der Gruppe < 50 nmol/l ergab eine Reduktion des Risikos, einen bösartigen Darmtumor zu entwickeln, von $72 \%$ !

Mitte 2008 folgte eine weitere Publikation aus den USA auf der Basis der „Nurses Health Study“ und der „Health Professional Follow up Study“. Hier wurden 304 Patienten mit einem bösartigen Darmtumor im Mittel 15 Jahre lang nach der Operation beobachtet. Die Einteilung in 2 Gruppen erfolgte nach der Höhe des Vitamin-D-Spiegels zum Zeitpunkt der Operation [22]. In der Gruppe mit den höheren Vitamin-D-Spiegeln reduzierte sich die Gesamtsterblichkeit um 48\%, die tumorbedingte Sterblichkeit um 39\%. Es finden sich somit deutliche Parallelen zu den Ergebnissen von Goodwin bei der Verlaufskontrolle von bösartigen Brusttumoren [7].

In dem schon erwähnten Artikel mit dem DINOMIT-Modell veröffentlichte die Arbeitsgruppe von Garland auch eine Weltkarte, die zum einen den in den einzelnen Ländern vorherrschenden VitaminD-Spiegel zeigt (Abb.4). Zum andern wird in den weiter nördlich/südlich gelegenen Breitengraden beider Hemisphären die zu erwartende Reduktion neu auftretender bösartiger Darmtumoren dargestellt, wenn der Vitamin-D-Spiegel durch die tägliche Gabe von 2000 Einheiten Vitamin D und/ oder eine regelmäßige Sonneneinwirkung angehoben würde [6].

Die Ergebnisse der Untersuchungen von Patienten mit Kolonkarzinomen sind somit nicht weniger eindrucksvoll als die 


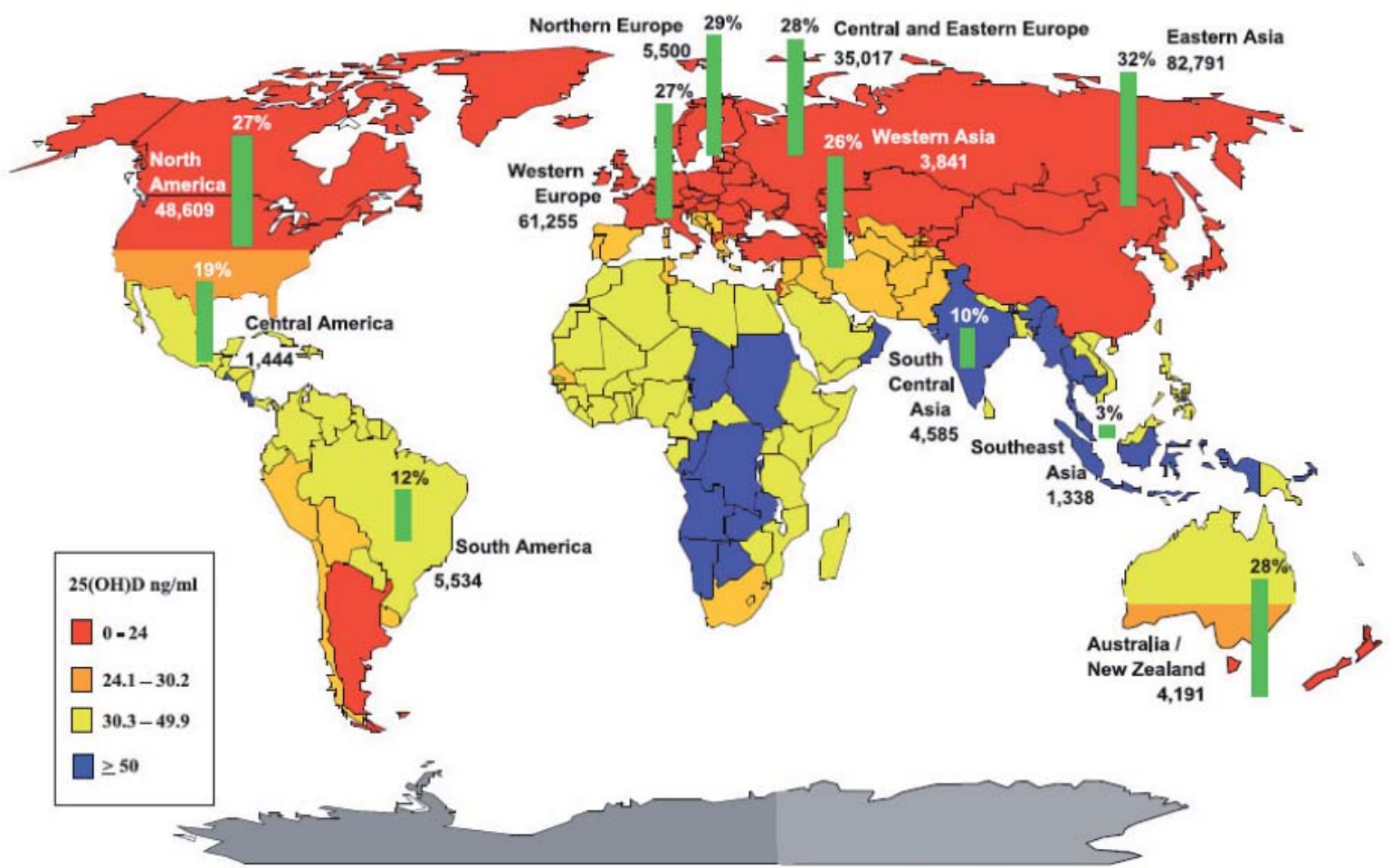

Abb. 4: Weltkarte mit den in einzelnen Ländern vorherrschenden Vitamin-D-Spiegeln und Prozentangabe der zu erwartenden Reduktion in dem jeweiligen Land für neu auftretende bösartiger Darmtumoren, wenn der Vitamin-D-Spiegel durch die Gabe von 2000 Einheiten Vitamin D und/ oder eine regelmäßige Sonneneinwirkung angehoben würde (mit freundlicher Genehmigung aus [6]).

Befunde bei den Patientinnen mit einem bösartigen Brusttumor. Eine Vielzahl weiterer bösartiger Tumore zeigt ebenfalls einen positiven, jedoch zum Teil noch weniger umfangreich dokumentierten Einfluss von Vitamin D. Die Tumore folgender Organe wurden untersucht: Blase, Lunge, Magen, Niere, Bauchspeicheldrüse und Speiseröhre. Auch für mehrere Blutkrebse (Leukämie, Multiples Myelom und Hodgkin-Lymphom) wird ein positiver Einfluss von Vitamin D diskutiert.

Ähnlich wie beim Mammakarzinom finden sich auch für die Kolonkarzinome inzwischen umfangreiche Meta-Analysen. So zeigt die EPIC-Studie (European Prospective Investigation into Cancer and Nutrition) mit 520.000 Teilnehmern aus zehn Ländern Westeuropas eine deutliche, vom Vitamin-D-Spiegel abhängige Risikosteigerung für bösartige Kolontumore [16]. Eine Meta-Analyse mit 35 voneinander unabhängigen, prospektiven Studien bestätigt die inverse Beziehung zwischen der Höhe des Spiegels des Sonnenhormons und dem Mortalitätsrisiko [23].

\section{Fazit}

Zum Abschluss der Vorstellung der Studienergebnisse mit Vitamin $\mathrm{D}$ bei speziellen Tumoren sei noch eine Mitte 2014 erschienene Arbeit von Mitarbeitern des Deutschen Krebsforschungszentrums in Heidelberg zitiert [20]. Es handelt sich um eine Meta-Analyse, die nochmals die Situation beim Mamma- und Kolonkarzinom untersucht. Dabei wurden jeweils fünf Studien mit 2330 Fällen von kolorektalen Tumoren und 4413 Fällen von Mammakarzinomen in Bezug auf die Mortalität in Abhängigkeit von der Höhe des Vitamin-D-Spiegels verglichen. Für die Patienten mit einem kolorektalen Karzinom berechnete sich bei ausreichendem Vitamin-D-Spiegel eine mittlere Risikoreduktion um $29 \%$ für die allgemeine Sterblichkeit und $35 \%$ für die tumorspezifische Sterblichkeit. Bei den Patientinnen mit einem Mammakarzinom ergaben sich jeweils $38 \%$ bzw. $42 \%$ Risikoreduktion.

In den Augen der Kritiker des neuen „Vitamin-D-Hype“ haben jedoch alle diese
Studien den Makel, dass sie einen niedrigeren Evidenzgrad haben, da sie nicht doppelblind, placebokontrolliert, randomisiert und prospektiv durchgeführt wurden. Doch auch dieses Argument lässt sich mit einer entsprechenden Studie entkräften. Zwar war der primäre Endpunkt der betreffenden Untersuchung nicht die Karzinomentstehung, sondern die Entwicklung einer Osteoporose, als weiterer Endpunkt wurde jedoch die Häufigkeit der Entstehung bösartiger Tumore in den untersuchten Gruppen miteinander verglichen.

Die Auswertung der Daten ergab, dass das allgemeine Tumorrisiko in der mit 1.100 IE Vitamin D täglich über vier Jahre behandelten Gruppe signifikant niedriger lag als in der Kontrollgruppe [18]. Das relative Risiko, unter dieser Vitamin-D-Zufuhr einen bösartigen Tumor zu entwickeln, betrug nur noch $40 \%$. Bereits diese Aussage ist sehr beachtlich! Wurden jedoch diejenigen Karzinome ausgeschlossen, die bereits im ersten Jahre auftraten und damit wahrscheinlich zu Beginn der Behandlung bereits vorhanden waren, er- 
gab sich eine echte Sensation: Das relative Risiko sank um 77\%! Die Kombination mit Kalzium zeigte dabei keine bessere Wirkung als Vitamin D alleine.

An der Wirksamkeit von Vitamin D auf die Entstehung und die Progredienz bösartiger Tumore besteht somit kein Zweifel, auch wenn die Ergebnisse nicht aller Studien konsistent sind und gebetsmühlenhaft immer wieder neue, prospektive Studien gefordert werden. Wer sich ungeachtet der Fülle dieser Informationen aus dem Bereich der Onkologie als Therapeut (noch) nicht durchringen kann, bei seinen Patienten (und sich selbst) das Vitamin-DDefizit zu beseitigen, der sollte sich zumindest nach den inzwischen international allseits anerkannten Vorgaben für die Therapie der Osteoporose richten. Hier wird ein Vitamin-D-Spiegel von mindestens $50 \mathrm{nmol} / \mathrm{l}$ gefordert. Selbst diesen (eigentlich überholten) Schwellenwert erreicht nämlich mehr als die Hälfte der deutschen Bevölkerung nicht! Wer also zumindest leitliniengemäß die Osteoporose seiner Patienten mit adäquaten Mengen von Vitamin D behandelt (Laborkontrollen!) bzw. einer Osteoporose damit vorbeugt, bekommt gratis und ohne Risiko ein ganzes Bündel von zusätzlichen Gesundheitseffekten - auch in Bezug auf das Karzinomgeschehen.
Autorenerklärung: Der Autor erklärt, dass er Honorare für Vorträge von diversen Vitamin-D-Produkte vertreibenden Firmen erhalten hat.

\section{online:}

http://dx.doi.org//10.1055/s-0033-1357713

\section{Korrespondenzadresse}

Prof. Dr. med. Jörg Spitz

Institut für Medizinische Information und

Prävention

Krauskopfallee 27

65388 Schlangenbad

E-Mail: info@mip-spitz.de

\section{Literatur}

[1] Abbas S, Linseisen J, Slanger T, Kropp S, Mutschelknauss EJ, Flesch-Janys D, ChangClaude J. Serum 25-hydroxyvitamin D and risk of post-menopausal breast cancer - results of a large case-control study. Carcinogenesis 2008; 29(1): 93-99

[2] Deutsche Gesellschaft für Ernährung (DGE). D A CH Referenzwerte für die Nährstoffzufuhr - Vitamin D. 1. Auflage, 4. korrigierter Nachdruck 2012. Hrsg. v. Österreichische Gesellschaft für Ernährung, Schweizerische Gesellschaft für Ernährungsforschung, Schweizerische Vereinigung für Ernährung, Deutsche Gesellschaft für Ernährung. Online verfügbar unter http://www.dge.de/pdf/ ws/Referenzwerte-2012-Vitamin-D.pdf, zuletzt aktualisiert am 10.01.2012, zuletzt geprüft am 28.05.2012

[3] Engel P, Fagherazzi G, Mesrine S, BoutronRuault MC, Clavel-Chapelon F. Joint effects of dietary vitamin $D$ and sun exposure on breast cancer risk: results from the French E3N cohort. Cancer Epidemiol Biomarkers Prev 2011; 20(1): 187-198

[4] Freedman DM, Looker AC, Chang SC, Graubard BI. Prospective study of serum vitamin $\mathrm{D}$ and cancer mortality in the United States. J Natl Cancer Inst 2007; 99(21): 1594-1602

[5] Garland CF, Gorham ED, Mohr SB, Grant WB, Giovannucci EL, Lipkin M et al. Vitamin D and prevention of breast cancer: pooled analysis. J Steroid Biochem Mol Biol 2007; 103(3-5): 708-711

[6] Garland CF, Gorham, ED, Mohr SB, Garland FC. Vitamin D for cancer prevention: global perspective. Ann Epidemiol 2009; 19(7): 468-483

[7] Goodwin PJ, Ennis M, Pritchard KI, Koo J, Hood N. Frequency of vitamin D (Vit D) deficiency at breast cancer (BC) diagnosis and association with risk of distant recurrence and death in a prospective cohort study of T1-3, N0-1, M0 BC. American Society of Clinical Oncology (2008 Annual Meeting, 26)

[8] Gorham ED, Garland CF, Garland FC, Grant WB, Mohr SB, Lipkin M, Newmark HL, Giovannucci E, Wei M, Holick MF. Optimal vitamin D status for colorectal cancer prevention: a quantitative meta analysis. Am J Prev Med 2007; 32(3): 210-216 
[9] Gröber U et al. Vitamin D: Update 2013. Von der Rachitis-Prophylaxe zur allgemeinen Gesundheitsvorsorge. Deutsche Apotheker Zeitung 2013; 153(15): 3000-3007.

[10] Heaney RP, Holick MF. Why the IOM recommendations for vitamin $\mathrm{D}$ are deficient. J Bone Miner Res 2011; 26(3): 455-7

[11] Hintzpeter B, Mensink GBM, Thierfelder W, Müller MJ, Scheidt-Nave C. Vitamin D status and health correlates among German adults. Eur J Clin Nutr 2007; 62(9): 1079-1089

[12] Hintzpeter B, Scheidt-Nave C, Müller M], Schenk L, Mensink GBM. Higher prevalence of vitamin $D$ deficiency is associated with immigrant background among children and adolescents in Germany. J Nutr 2008; 138 (8): 1482-1490

[13] Holick MF. Vitamin D: Sonne, Quellen und die „D-batte“. In: Reichrath J, Lehmann B, Spitz J, Hrsg. Vitamin D Update 2012. Von der Rachitisprophylaxe zur allgemeinen Gesundheitsvorsorge. München-Deisenhofen: Dustri-Verl. Feistle; 2012: 61-88

[14] Holick MF, Binkley NC, Bischoff-Ferrari HA, Gordon CM, Hanley DA, Heaney RP et al. Evaluation, treatment, and prevention of vitamin D deficiency: an Endocrine Society clinical practice guideline. J Clin Endocrinol Metab 2011; 96(7): 1911-1930

[15] Hollis BW, Wagner CL. Vitamin D requirements and supplementation during pregnancy. Curr Opin Endocrinol Diabetes Obes 2011; 18(6): 371-5

[16] Jenab M, Bueno-de-Mesquita HB, Ferrari P, van Duijnhoven FJB, Norat T, Pischon T et al. Association between pre-diagnostic circulating vitamin $\mathrm{D}$ concentration and risk of colorectal cancer in European populations: a nested case-control study. BMJ 2010; 340: 5500

[17] John EM, Schwartz GG, Koo J, Wang W, Ingles SA. Sun exposure, vitamin $D$ receptor gene polymorphisms, and breast cancer risk in a multiethnic population. Am J Epidemiol 2007: 166 (12): 1409-1419

[18] Lappe JM, Travers-Gustafson D, Davies KM, Recker RR, Heaney RP. Vitamin D and calcium supplementation reduces cancer risk: results of a randomized trial. Am J Clin Nutr 2007; 85(6): 1586-1591

[19] Lin J, Manson JE, Lee IM, Cook NR, Buring JE, Zhang SM. Intakes of calcium and vitamin D and breast cancer risk in women. Arch Intern Med 2007; 167(10): 1050-1059

\section{Summary}

The formerly limited working range of vitamin D with regard to calcium metabolism has been extended to an improved function of nearly all cells and organs of our body demonstrating properties of a hormone. These include the modulation of roundabout 2000 genes. This information is based on the detection of vitamin D receptors almost everywhere in our body. Meanwhile even the exact signaling cascades of vitamin $D$ in the cells are known. Unfortunately the UVB depending production of vitamin D (the sunshine hormone) in our skin has become a problem due to several aspects of our modern lifestyle. By this not only people living in sun deprived countries are at high risk for vitamin D deficiency but also people living in sunny countries like Turkey, India or Australia. The need of vitamin D for a correct cell metabolism on the one side and the deficiency of a large part of the population on the other side are responsible for the promotion of the non-communicable diseases of civilization. Typically cancer development and progress are involved as well. The sunshine hormone promotes a suppression of tumor growth, attenuation of signals to produce metastasis, enhanced cell death (apoptosis), promotion of cell differentiation (in the direction of the benignity) as well as angiogenesis by the tumor. This is also true for two of the most prevalent kinds of carcinoma: breast cancer in women and: colon carcinoma of both sexes. A large number of epidemiologic and prospective studies document a risk reduction of 50-70 percent for tumor development and a similar risk reduction for mortality. So it seems advisable to take care to have a sufficient vitamin D level $(>30 \mathrm{ng} / \mathrm{ml})$ to enjoy the positive effects of the sunshine hormone. This in not only related to bone metabolism but also to all the other positive effects in our body - cancer suppression included.

Keywords: Vitamin D, sunshine hormone, endocrine function, properties in oncology, colon cancer, breast cancer

[20] Maalmi H, Ordonez-Mena JM, Schottker B, Brenner H. Serum 25-hydroxyvitamin D levels and survival in colorectal and breast cancer patients: systematic review and meta-analysis of prospective cohort studies. Eur J Cancer 2014; 50(8): 1510-1521

[21] Mohr SB, Gorham ED, Kim J, Hofflich H, Garland CF. Meta-analysis of vitamin D sufficiency for improving survival of patients with breast cancer. Anticancer Res 2014; 34(3): 1163-1166

[22] Ng K, Meyerhardt JA, Wu K, Feskanich D, Hollis BW, Fuchs CS. Circulating 25-hydroxyvitamin d levels and survival in patients with colorectal cancer. J Clin Oncol 2008; 26(18): 2984-2991

[23] Pilz S, lodice S, Zittermann A, Grant WB, Gandini S. Vitamin D status and mortality risk in CKD: a meta-analysis of prospective studies. Am J Kidney Dis 2011; 58(3): 374382
[24] Robien K, Cutler G], Lazovich D. Vitamin D intake and breast cancer risk in postmenopausal women: the lowa Women's Health Study. Cancer Causes Control 2007; 18(7): 775-782

[25] Spitz J. Vitamin D. Das Sonnenhormon für unsere Gesundheit und der Schlüssel zur Prävention. 2., erw. Aufl. Schlangenbad: Ges. für Medizinische Information und Prävention; 2009

[26] Spitz J, Grant WB. Krebszellen mögen keine Sonne. Vitamin D - der Schutzschild gegen Krebs, Diabetes und Herzerkrankungen. Murnau a. Staffelsee: Mankau; 2010

[27] Vieth R, Bischoff-Ferrari H, Boucher B], Dawson-Hughes B, Garland CF, Heaney RP, Holick MF, Hollis BW, Lamberg-Allardt C, McGrath Jj, Norman AW, Scragg R, Whiting SJ, Willett WC, Zittermann A. The urgent need to recommend an intake of vitamin $D$ that is effective. Am J Clin Nutr 2007; 85(3): 649-650 